\title{
Modifications of functional respiratory tests in non-smoker patients with rheumatoid arthritis
}

\author{
1 "Ovidius" University of Constanta, Faculty of General Medicine, Discipline of Medical Semiology, Romania \\ ${ }^{2}$ Clinical Port Hospital of Constanta, IInd Medical Department Romania
}

\begin{abstract}
This paper aim is to study lower airways' disorders in non-smoker patients with rheumatoid arthritis (RA) without respiratory symptoms and to reveal the correlations between the lower airways' disorders on one side and RA dependent variables and demographic variables on the other side. Our study included 145 women diagnosed with RA. Airways evaluation was assessed by spirometry. Patients with pulmonary radiological modifications, pulmonary diseases or respiratory symptoms (cough, dyspnea) were not included in the study. Bivariate analysis was used to analyze the obtained variables. The medium age was $56.28 \pm 10.24$ years. The medium body mass index (BMI) was $27.66 \pm 3.23 \mathrm{~kg} / \mathrm{m} 2$. The modifications of respiratory functional tests revealed by spirometry were the following: $15.86 \%$ had FEV1/FVC $<70 \%, 32.41 \%$ of patients had FEF50<60\%, $57.93 \%$ of patients had FEV $1<80 \%$ and $66.89 \%$ of patients had $\mathrm{FCV}<80 \%$. The repartition according to RA stage was: $60.68 \%$ in the IInd stage, $31.03 \%$ in the IIIrd stage and $8.96 \%$ in the IVth stage. Spirometry evaluated disorders of the airways and pulmonary interstitium are frequently found in patients with RA, without symptoms and without radiological anomalies.
\end{abstract}

Keywords: rheumatoid arthritis, respiratory functional tests

Claudia Mihailov

B-dul 1 Mai nr 5-7, Constanţa, România ;

Tel: +40-0241-583-713

E-mail: claudiamihailov@yahoo.com

\section{Introduction}

Lung involvement in patients with rheumatoid arthritis (RA) is frequent, most of the time symptomless. Among RA, other factors involved in pulmonary disease are: DMARS, infections due to immunosuppression and coexistent medical disorders (COPD, asthma, heart failure).

RA affects mostly the pulmonary interstitium, but the airways and pleura also (1). The prevalence of distal bronchi obstruction in non-smoker patients, identified through functional respiratory tests varies between 0 and $24 \%(2,3)$ and rises up to $60 \%$ if high resolution pulmonary CT (HRCT) is used to detect obstruction (3).

This paper aim is to study the lower airways disorder in patients with RA and to reveal the correlations between the modifications of respiratory functional tests on one side and RA variables and demographic variables on the other side.

\section{Material, method and patients}

One hundred forty-five consecutive patients of the Rheumatology Department of the Clinical Railway Hospital of Constanta were included in the 
study. All patients fulfilled the ACR 1987 revised for RA criteria. Partial results of this study were published in 2010 (4).

Demographical variables, those related to RA and to patients' therapy (Table no.1) were obtained by interview. The following data was collected: personal pathological history, exposure to pulmonary pollutants, age, weight, height, BMI, smoker or not, stage of RA, age at RA onset, duration of RA, the presence of rheumatoid factor (RF), erythrocyte sedimentation rate (ESR), $\mathrm{C}$ reactive protein (CRP), disease modifying antirheumatic drugs (DMARD).

Table no. 1: Group's features

\begin{tabular}{|l|c|}
\hline \multicolumn{2}{|c|}{ GROUP'S FEATURES (N=145) } \\
\hline Age (years) & $56,28 \pm 10,24$ \\
\hline Height $(\mathrm{cm})$ & $160,21 \pm 6,26$ \\
\hline Weight $(\mathrm{kg})$ & $71 \pm 12,68$ \\
\hline Body Mass Index $(\mathrm{kg} / \mathrm{m} 2)$ & $27,66 \pm 3,23$ \\
\hline FCV $\%$ & $81,20 \pm 26,52$ \\
\hline FEV1, \% & $78,18 \pm 16,03$ \\
\hline FEV1/FCV, \% (\%) & $83,58 \pm 16,20$ \\
\hline FEF50, $\%$ & $70,28 \pm 20,15$ \\
\hline RA duration & $8,18 \pm 7,92$ \\
\hline RA stage & \\
\hline STAGE 2 $(\%)$ & $60,68 \%(88)$ \\
\hline STAGE 3 $\%)$ & $31,03 \%(45)$ \\
\hline STAGE 4 $(\%)$ & $8,96 \%(13)$ \\
\hline POZITIVE RF $(\%)$ & $69,65 \%(101)$ \\
\hline ESR $(\mathrm{mm} / \mathrm{h})$ & $45,18 \pm 18,20$ \\
\hline CRP & $12,45 \pm 10,18$ \\
\hline M-HAQ & $1,68 \pm 0,51$ \\
\hline DAS & $4,53 \pm 1,4$ \\
\hline TREATMENT & \\
\hline METHOTREXATE & $45 \%$ \\
\hline SULPHASALASINE & $10 \%$ \\
\hline LEFLUNOMIDE & $49 \%$ \\
\hline BILOGICAL THERAPY & $6 \%$ \\
\hline
\end{tabular}

Global assessment of the disease was performed by measuring on a visual analog scale (VAS) from 0 to $100 \mathrm{~mm}$. Joint evaluation consisted in countering the painful joints (28 joints) and swollen joints (28 joints). Disease activity score 28 (DAS 28) was measured using the 28 joints, VAS and ESR.

Physical disability was assessed by means of modified Health Assessment Questionnaire, (M-HAQ). (5)

Patients were considered seropositive if RF titer was $\geq 64 \mathrm{iu} / \mathrm{ml}$ at least in one determination.

All patients had a chest X Ray interpreted by an experimented radiologist.

Respiratory functional tests were performed with a spirometer (Chest Graph HI-105). The following parameters were measured: forced vital capacity (FVC), forced expired volume in one second (FEV1), forced expiratory flow at $50 \%$ of vital capacity (FEF50), FEV1/FCV ratio. Results were registered only if expire was correctly done. Both for FEV1 and for FCV the highest values of three consecutive measurements with a difference of less than $5 \%$ or $100 \mathrm{ml}$ between the 3 curves were considered. FEV1/ FCV ratio was calculated The FEV1/FCV ratio was realized using the biggest values of FEV1 and FCV from the technical curves (not necessarily the same curve). FCV, FEV1 and FEF50 were expressed as percentage from the predicted values. Patients were classified as having large airways obstruction if FEV1/FCV was smaller than $70 \%$ and small airways obstructions if FEF50 was less than $60 \%$. We used the American Medical Association classification, (AMA), to classify improper FCV and FEV1.

Criteria for exclusion from the study were:

- smoker patients

- patients with diseases that could determine ventilatory dysfunction: heart disease (heart failure), pulmonary disease (COPD, bronchial asthma, interstitial pulmonary disease, acute pulmonary infections), thoracic deformities (e.g.: deformities due to osteoporotic vertebral fractures;

- patients in whom chest X-ray revealed cardiomegaly and/or pulmonary disorders

We used bivariate analyses to compare demographic variables and those RA related in patients with and without ventilatory dysfunction. In order to compare normal distributed continuous variables 
"t student " test was used, while for the continuous variable with non-normal distribution Mann-Whitney $U$ test was used, for dichotomic variables $\chi^{2}$ test was used. $\mathrm{P}$ was considered significant at a value less than 0.05 .

Statistic analysis was performed on a computer program (MedCal for Windows 9th version).

\section{Results and Discussion}

Features of the studied group were presented in Table no.1.

The medium age of the patients was $56.28 \pm 10.24$ years, the repartition on age groups is featured in (Figure 1): $3862 \%$ of patients under 50 years old, $42.75 \%$ between $50-60$ years and $18.6 \%$ over 60 years old. The medium body mass index (BMI) was $27.66 \pm 3.23 \mathrm{~kg} / \mathrm{m} 2$

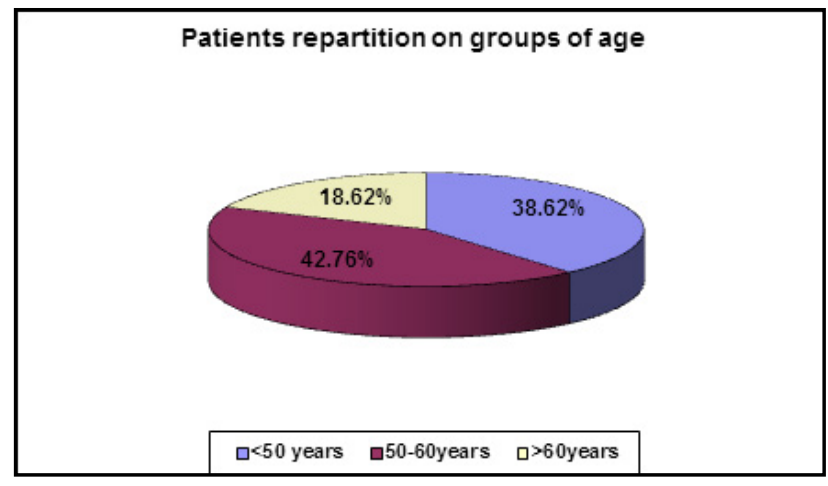

Figure no 1. Patients repartition on groups of age

The modifications of respiratory functional tests revealed by spirometry were the following: $15.86 \%$ had FEV $1 / \mathrm{FVC}<70 \%, 32.41 \%$ of patients had FEF $50<60 \%, 57.93 \%$ of patients had FEV $1<80 \%$ and $66.89 \%$ of patients had FCV $<80 \%$ (Figure 2). FCV was normal in $33.1 \%$ patients, $47.36 \%$ of patients had FCV between $60-79 \%$ from the predicted value, in $16.55 \%$ of the patients FEV1 was $51-59 \%$ from the predicted value while in $2.78 \%$ of patients FCV was $<50 \%$ from the predicted value (Figure 3 ). As far as FEV1, in $42.07 \%$ of the patients FEV1 was within normal limits, $48.27 \%$ had a FEV1 between $60-79 \%$ from the predicted value, $6.2 \%$ from the patients had FEV1 between $51-59 \%$ from the predicted value and in $3.44 \%$ from the patients FCV was less than $50 \%$ from the predicted value (Figure no.3)

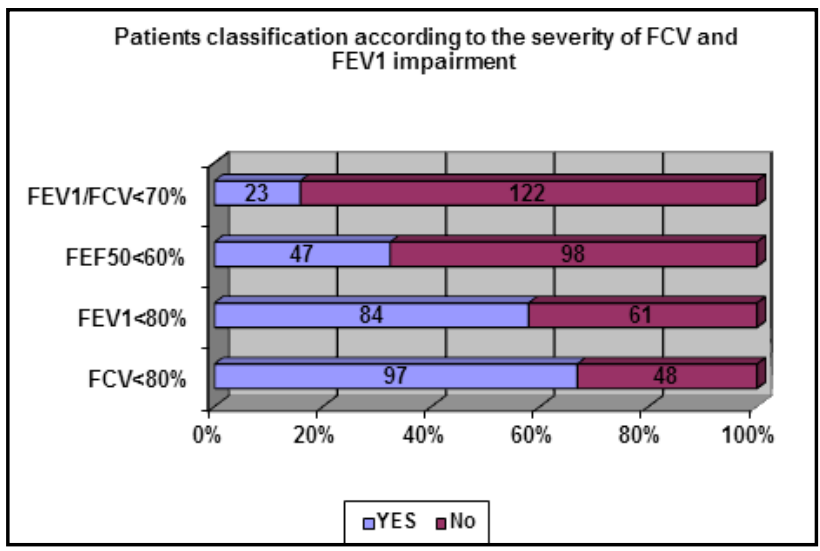

Figure no 2. Patients repartition according to the presence of respiratory tests modifications

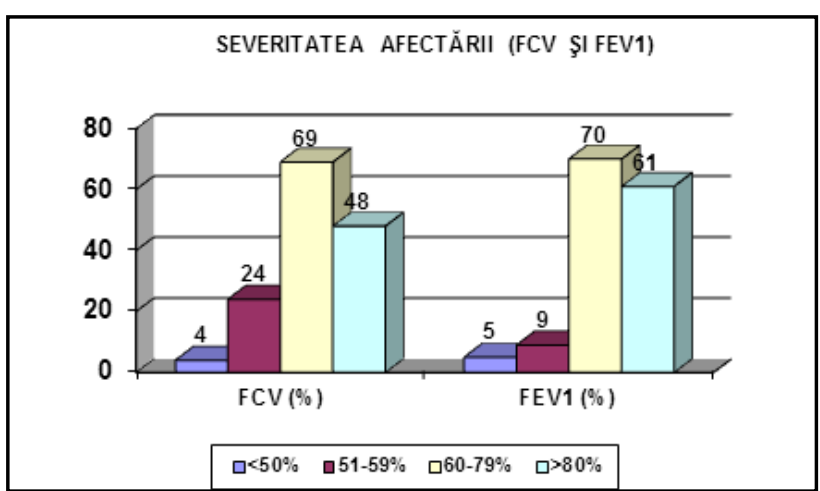

Figure no.3. Patients classification according to the severity of FCV and FEV1 impairment

The medium duration of RA was $8,19 \pm 7,92$ years. Most of the patients were in the IInd stage $(60.68 \%), 31.03 \%$ were in the IIIrd stage and only $8.96 \%$ of the patients were in the IVth stage (Figure 4). Rheumatoid factor was positive in $69.65 \%$ of the patients. Medium ESR was $45.18 \pm 18.20 \mathrm{~mm} / \mathrm{h}$, and CRP level was $12.45 \pm 10,18 \mathrm{mg} / \mathrm{dl}$. M-HAQ score had a medium value of $1,68 \pm 0,51$, while DAS 28 score of $4.53 \pm 1.4$. 
DMARD (disease modifying anthirheumatic drugs) received by the patients was: $6 \%$-biological therapy, $10 \%$ sulphasalazine, $45 \%$ methotrexate and $49 \%$ leflunomide.

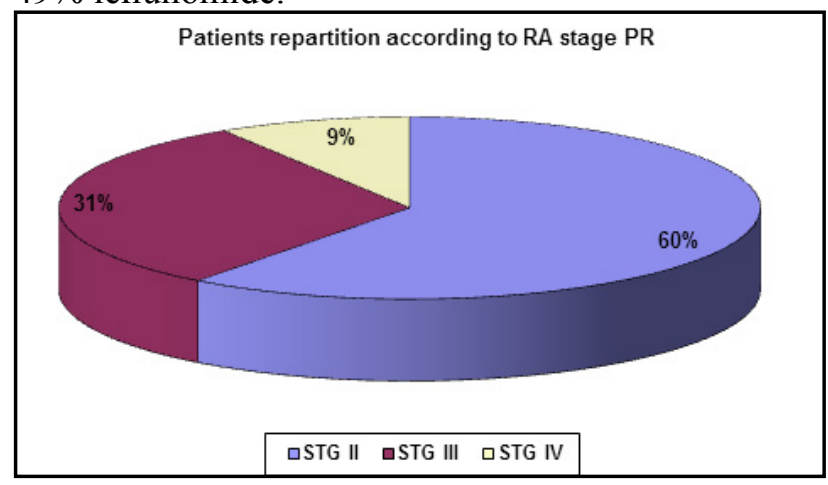

Figure no 4. Patients repartition according to RA stage PR

We compared patients with and without ventilatory dysfunction, and no association between ventilatory dysfunction and demographical variables or RA related variables was revealed.

The high prevalence of obstructive and restrictive ventilatory dysfunction in our study is the same as other studies from the literature, studies which found a high prevalence of the respiratory dysfunction.

In our study we did not find correlations between the anomalies of the functional respiratory tests and the variables related to RA or demographical variables. In the study of Vergnenegre and co. (6) an association was revealed between the obstruction of distal bronchi - evaluated by FEF25-75 and the duration of RA. In another study, of Thierry şi co. (3), correlations between distal airways obstruction on one side and high resolution CT revealed bronchiectasis and bronchial infections, on the other side, were found. But no correlation between distal airway obstruction and RA related variables were detected. There were also patients in which spirometry was normal and high resolution pulmonary $\mathrm{CT}$ revealed lesions of distal bronchi, secondary to RA.

\section{Conclusions}

- Pulmonary disease in non-smoker patients with RA, with no radiological pulmonary modifications is frequent and symptomless.

- Ventilatory dysfunction: obstructive or restrictive, in patients with RA, is usually mild.

- The prevalence of distal airways obstruction was $34.41 \%$ and in large airways was $15.86 \%$.

- No correlations were found between the modifications of functional respiratory tests and RA dependent variables.

\section{References}

1. Tanoue LT. (1998). Pulmonary manifestations of rheumatoid arthritis. Clin Chest Med. 19(4), 667-85.

2. Sassoon CS, McAlpine SW, Tashkin DP, Baydur A, Quismorio FP. \& Mongan ES. (1984). Small airways function in nonsmokers with rheumatoid arthritis. Arthritis Rheum. 27(11), 1218.

3. Perez T, Remy-Jardin M. \& Cortet B. (1998). Airways involvement in rheumatoid arthritis. Clinical, functional, and HRCT findings. $\mathrm{Am} \mathrm{J}$ Respir Crit Care Med. 157(5 Pt 1), 1658.

4. Claudia Ileana Mihailov, A.N. Mitroi \& Irina Tica. (2010). Modifications of functional respiratory tests in non-smoker patients with rheumatoid arthritis. Archives of Balkan Medical Union. 45(4), 286-289.

5. Pincus T, Summey JA., Soraci SA Jr, Wallston KA \& Hummon NP. (1983). Assessment of patient satisfaction in activities of daily living using a modified Stanford Health Assessment Questionnaire. Arthritis Rheum. 26, 1346-1353.

6. Vergnenegre A, Pugnere N, Antonini MT, Arnaud M, Melloni B. \& Treves R. et al. (1997). Airway obstruction and rheumatoid arthritis. Eur. Eur Respir J. 10, 1072-1078. DOI: 10.1183/09031936.97.10051072. 